\title{
Correspondence
}

\section{Abbreviated identification of Candida albicans by the presence of a pseudohyphal fringe ('spiking' appearance) - some caveats}

Candida albicans is the most commonly encountered yeast pathogen in our clinical laboratory. Workers such as Barnes \& Vale (2005) and Nagaishi \& Baron (1997) have described the rapid identification of $C$. albicans solely based on the presence of a pseudohyphal fringe ('spiking' colony morphology) with excellent sensitivity (even surpassing that of the wellestablished germ-tube test) and specificity. This method is also supported by the Clinical and Laboratory Standards Institute M35-A2 document 'Abbreviated Identification of Bacteria and Yeast' (CLSI, 2008), which states that colonies growing for less than $48 \mathrm{~h}$ on blood-containing medium that exhibit mycelial 'star-like' or 'feet' projections into the agar may be identified as $C$. albicans. The use of an obvious morphological feature to immediately identify C. albicans is of great interest to us as it would save considerable time, effort and cost, and potentially translate to more timely information being conveyed to the clinician.

We therefore evaluated the performance of this method using our standard trypticase soy agar with $5 \%$ sheep blood (TSA-BAP), and following overnight (18-24 h) incubation in a $\mathrm{CO}_{2}$ incubator, using our standard germ-tube method with horse serum as the reference standard. A positive germ-tube test result was taken to indicate C. albicans/Candida dubliniensis (separation of these species requires additional testing). Germ-tube-negative isolates underwent further testing including urease testing and examination for chlamydospore production on cornmeal agar. The incubation time of 18 $24 \mathrm{~h}$ was determined by our workflow, corresponding to the initial reading of the plate on the day after inoculation.

A total of 112 consecutive non-pigmented yeast isolates from various clinical specimens (blood, peritoneal, wound, lines, urine, etc.) received by our laboratory for routine culture, between July 2009 and November 2009, were included. Forty-two isolates were germ-tube positive, and hence were presumptively identified as $C$. albicans/C. dubliniensis. Of these 42 germtube positive isolates, only 33 showed the presence of a pseudohyphal fringe ('spiking'), yielding a sensitivity of $78.6 \%$ for the observation of 'spiking' in $C$. albicans/C. dubliniensis. Of the 70 germtube negative yeast isolates, 67 did not show 'spiking', yielding a specificity of $95.7 \%$. The overall inter-test agreement was good (Cohen's $\kappa=0.76$ ). These results are summarized in Table 1. Interestingly, the three germ-tube negative isolates that demonstrated pseudohyphal 'spiking' showed the presence of arthroconidia (in addition to blastoconidia) on microscopic (Gram-stain) examination, and further phenotypic workup confirmed these isolates to be Trichosporon sp. rather than $C$. albicans.

Even though we found this rapid method of morphological identification of $C$. albicans/C. dubliniensis to be less sensitive than previously described, it appears to be feasible to use it for the presumptive identification of yeasts for semi-critical and non-sterile sites. It is, however, crucial to note that non-Candida yeasts (such as Trichosporon spp.) may give false-positive results for 'spiking', as in our study. Although the presence of a pseudohyphal fringe in colonies of Trichosporon spp. has been recognized previously (www. mycology.adelaide.edu.au - Mycology Online, University of Adelaide, Australia), the possibility of mistaking such colonies for 'spiking' colonies of C. albicans, to the best of our knowledge, has not been described. As the susceptibility profiles of these two genera of yeasts may differ significantly (Pfaller \& Diekema, 2004), it would be crucial for the clinical laboratory to differentiate between the two, especially when the isolate is derived from a sterile site or suspected to be causing a more serious illness. We therefore suggest that the Gram-stain morphology of any 'spiking' isolate be carefully examined for the presence of arthroconidia (which are indicative of Trichosporon spp.).

Additional analysis, such as urease testing, may be indicated in suspicious cases.

Table 1. Comparison of the performance of pseudohyphal fringe ('spiking') appearance at $18-24 \mathrm{~h}$ incubation on $5 \%$ TSA-BAP in $\mathrm{CO}_{2}$ to the germ-tube test using horse serum in identifying C. albicans/C. dubliniensis

Sensitivity of 'spiking' in comparison to the germ-tube test $=33 / 42=78.6 \%$; specificity of 'spiking' in comparison to the germ-tube test $=$ $67 / 70=95.7 \%$; positive predictive value of 'spiking' in comparison to germ-tube test $=33 / 36=91.7 \%$; negative predictive value of 'spiking' in comparison to germ-tube test $=67 / 76=88.2 \%$; overall inter-test agreement (Cohen's $\kappa)=0.76(0.64-0.89)$.

\begin{tabular}{|c|c|c|c|}
\hline \multirow[t]{2}{*}{ Pseudohyphal fringe ('spiking') } & \multicolumn{2}{|c|}{ Germ tube } & \multirow[t]{2}{*}{ Total } \\
\hline & Positive $(+)$ & Negative $(-)$ & \\
\hline Absent $(-)$ & 9 & 67 & 76 \\
\hline Total & 42 & 70 & 112 \\
\hline
\end{tabular}


There are some limitations to our study. The lower sensitivity observed in our study may possibly be due to the use of an abridged incubation time of 18-24 h, as compared to the 24-48 $\mathrm{h}$ described in other studies (Barnes \& Vale, 2005; Nagaishi \& Baron, 1997). However, a shorter incubation period may be more realistic in the clinical laboratory setting, where clinicians may expect quicker delivery of preliminary information to guide patient management. Furthermore, adoption of this method by our laboratory would result in a significant reduction in our germ-tube testing volume, translating into savings in terms of labour and cost of reagents.

Neither the pseudohyphal fringe nor the germ-tube test allows confident separation between C. albicans and C. dubliniensis. For instance, Barnes \& Vale (2005) noted that $20 \%$ of C. dubliniensis isolates produced 'spiking' colonies and $40 \%$ showed positive germ-tube test results. We cannot determine what proportion of our study isolates were C. dubliniensis; however, recent susceptibility data from Pfaller et al. (2010) seem to suggest that the antifungal susceptibility profile $C$. dubliniensis is comparable with that of $C$. albicans. Further testing to separate these species may be indicated for critical infections.

\section{Kian Sing Chan, Rama Narayana Deepak, Mei Gie Tan and Ai Ling Tan}

Department of Pathology, Singapore General Hospital, Singapore

Correspondence: Kian Sing Chan (chankiansing@yahoo.com.sg)

Barnes, R. A. \& Vale, L. (2005). 'Spiking' as a rapid method for differentiation of Candida albicans from other yeast species. J Hosp Infect 60, 78-80.

CLSI (2008). Abbreviated Identification of Bacteria and Yeast, approved guidelines, 2nd edn, p. 18, M35-A2. Wayne, PA: Clinical and Laboratory Standards Institute.

Nagaishi, K. \& Baron, E. J. (1997). Identification of Candida albicans by colony morphology. Clin Microbiol Newsl 19, 112.

Pfaller, M. A. \& Diekema, D. J. (2004). Rare and emerging opportunistic fungal pathogens: concern for resistance beyond Candida albicans and Aspergillus fumigatus. J Clin Microbiol 42, 4419-4431.

Pfaller, M. A., Diekema, D. J., Gibbs, D. L., Newell, V. A., Ellis, D., Tullio, V., Rodloff, A., Fu, W., Ling, T. A. \& the Global Antifungal Surveillance Group (2010). Results from the ARTEMIS DISK global antifungal surveillance study, 1997 to 2007: a 10.5-year analysis of susceptibilities of Candida species to fluconazole and voriconazole as determined by CLSI standardized disk diffusion. J Clin Microbiol 48, 1366-1377. 